\title{
Le paramètre pression : un outil pour l'optimisation des matériaux piézo-électriques
}

\author{
J. Haines, J. Rouquette, V. Bornand, M. Pintard et Ph. Papet \\ Laboratoire de Physico-Chimie de la Matière Condensée, UMR CNRS 5617, Université \\ Montpellier II Sciences et Techniques du Languedoc, cc 003, Place Eugène Bataillon, \\ 34095 Montpellier Cedex 5, France
}

\begin{abstract}
Résumé. Le paramètre pression modifie de façon très importante la structure cristalline des solides. Ce paramètre peut induire des variations structurales continues sans augmenter l'agitation thermique et présente donc des avantages par rapport aux paramètres température et composition. L'effet de la pression est de réduire le volume. Cela se fait par: diminution des distances interatomiques, variations angulaires, rotation de polyèdres, transitions structurales avec dans de nombreux cas une augmentation de la coordinence des cations et une modification de la nature des liaisons chimiques. Les propriétés physiques du matériau se modifient en fonction des variations de la structure cristalline. Ainsi, en reproduisant les conditions thermodynamiques auxquelles sont soumis, naturellement ou volontairement, les matériaux, le paramètre pression nous fournit un outil puissant pour la conception et l'optimisation des dispositifs où ils s'intègrent. Les solutions solides $\mathrm{PbZr}_{1-\mathrm{x}} \mathrm{Ti}_{\mathrm{x}} \mathrm{O}_{3}$ (PZT) sont à la base des matériaux piézo-céramiques les plus utilisés à l'heure actuelle. Leurs propriétés diélectriques et piézoélectriques sont très sensibles aux contraintes dues aux champs élastiques ou électriques. Il est donc important de connaître l'effet de la pression hydrostatique sur la nature et la stabilité des phases de ce système. Nos résultats, obtenus par diffraction des rayons $\mathrm{X}$, diffraction neutronique, diffusion Raman et mesures diélectriques sous pression, montrent que la phase monoclinique $\mathrm{F}_{\mathrm{M}}{ }^{\mathrm{HT}}$, qui est à l'origine des propriétés piézoélectriques remarquables de la composition morphotropique $\left(\mathrm{PbZr}_{0,52} \mathrm{Ti}_{0,48} \mathrm{O}_{3}\right)$ à pression ambiante, peut également être obtenue à des pressions modérées dans la zone riche en $\mathrm{PbTiO}_{3} \mathrm{du}$ diagramme de phases. D'où la notion nouvelle «d'adaptabilité » de la solution solide introduite par la variable pression. Le diagramme de phases $\mathrm{P}-\mathrm{x}$ obtenu à partir de ces résultats devrait nous permettre de mieux comprendre les effets des contraintes sur les propriétés de ces matériaux et par conséquent d'optimiser leur élaboration pour des applications sous fortes contraintes.
\end{abstract}

\section{INTRODUCTION}

\subsection{Le paramètre pression}

La pression $(P)$ est une variable thermodynamique intensive comme la température $(T)$. Ce paramètre est conjugué avec le volume $(V)$, qui dérive de l'enthalpie libre $(G)$ par différentiation :

$$
V=\left(\frac{\partial G}{\partial P}\right)_{T}
$$

La dérivée seconde de $G$ permet d'accéder à la compressibilité $(k)$ :

$$
k=-\frac{1}{V}\left(\frac{\partial V}{\partial P}\right)_{T}
$$


Le comportement de $G$ en fonction de $P$ pour une transition du premier ordre est donné sur la figure 1 où la pente de la courbe correspond au volume et la dérivée seconde est égale à $-k V$. La relation entre $P$ et $V$ peut être décrite à l'aide d'une équation d'état [1]. Celle de Birch-Murnaghan [2], basée sur le modèle d'une déformation finie (développement de l'énergie libre en fonction de la déformation finie limité au $2^{\text {ème }}, 3^{\text {ème }}$ ou $4^{\text {ème }}$ ordre, exemple ci-dessous), est parmi les plus utilisées :

$$
\begin{gathered}
P=\frac{3}{2} B_{0}\left[\left(\frac{V}{V_{0}}\right)^{\frac{-7}{3}}-\left(\frac{V}{V_{0}}\right)^{\frac{-5}{3}}\right] \\
{\left[1+\frac{3}{4}\left(B_{0}^{\prime}-4\right)\left(\left(\frac{V}{V_{0}}\right)^{\frac{-2}{3}}-1\right)+\frac{3}{8}\left(B_{0} B_{0}^{\prime \prime}+\left(B_{0}^{\prime}-4\right)\left(B_{0}^{\prime \prime}-3\right)+\frac{35}{9}\right)\left(\left(\frac{V}{V_{0}}\right)^{\frac{-2}{3}}-1\right)^{2}\right]}
\end{gathered}
$$

où $B_{0}$ représente le module de compression $(B=1 / k)$, et $B_{0}^{\prime}$ et $B^{\prime \prime}{ }_{0}$ sont respectivement les dérivées premières et secondes de $B$ en fonction de $P$ à $P=0$.

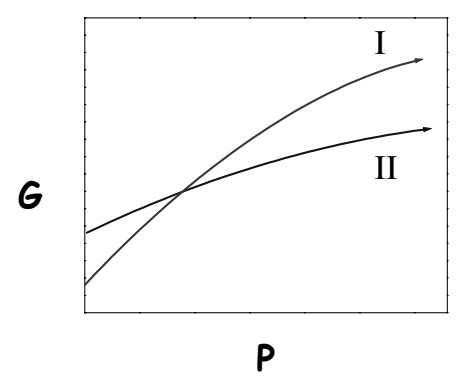

Figure 1. Enthalpie libre en fonction de la pression : cas d'une transition du $1^{\mathrm{er}}$ ordre (phases I, II).

Au niveau fondamental, les études sous pression permettent de déterminer des diagrammes de phases binaires pression-température et pression-composition. On peut également obtenir des valeurs thermodynamiques $\left(B_{0}, B_{0}^{\prime}, B^{\prime \prime}{ }_{0}\right.$, etc.). Dans le domaine des sciences des matériaux, le paramètre pression modifie de façon très importante la structure cristalline des solides. Ce paramètre peut induire des variations structurales continues sans augmenter l'agitation thermique et présente donc des avantages par rapport aux paramètres température et composition. L'effet direct de la pression est de réduire le volume par diminution des distances interatomiques, variations angulaires, rotation de polyèdres et/ou apparition de nouvelles transitions de phases. Ces transitions s'accompagnent souvent d'une augmentation de la coordinence (exemple : transition graphite-diamant), de changements de la nature de la liaison chimique (polymérisation de l'éthylène, acétylène, benzène etc., métallisation de $\mathrm{Si}, \mathrm{ZnS}, \mathrm{CsI}, \mathrm{O}_{2}, \mathrm{I}_{2}$, Xe etc.) et même de processus de décomposition chimique. Bien évidemment, les propriétés physiques du matériau se modifient en fonction des variations de la structure cristalline. Cela nous fournit un outil puissant pour la conception et l'optimisation des matériaux.

\subsection{Les solutions solides PbZr1-xTixO3 (PZT) - l'état de l'art}

Les solutions solides $\mathrm{PbZr}_{1-\mathrm{x}} \mathrm{Ti}_{\mathrm{x}} \mathrm{O}_{3}$ (PZT) sont à la base des matériaux piézo-céramiques les plus utilisés à l'heure actuelle. Ils se présentent sous forme massive comme sous forme de films minces où les niveaux de contraintes pour ces derniers peuvent atteindre voire dépasser les quelques gigapascals (GPa). Or, il est parfaitement établi que pour ce type de matériau, des champs élastiques ou électriques suffisamment intenses peuvent induire des modifications profondes de la structure cristallographique du matériau et en conséquence de ses propriétés diélectriques et piézo-électriques. 
Il est donc important de connaître l'effet de la pression sur la nature et la stabilité des phases de ce système. Ces matériaux de structure pérovskite présentent deux types d'instabilité : des déplacements ferroélectriques et des rotations des octaèdres $(\mathrm{Zr}, \mathrm{Ti}) \mathrm{O}_{6}$. Cela est à l'origine du diagramme de phases très riche de $\mathrm{PbZr}_{1-\mathrm{x}} \mathrm{Ti}_{\mathrm{x}} \mathrm{O}_{3}$. Depuis plus de trente ans, les propriétés piézoélectriques remarquables des solutions solides PZT ont été attribuées à un mélange de phases quadratique $\left(F_{Q}\right)$ et rhomboédrique $\left(F_{R}\right)$ près de la frontière morphotropique (transition de phases en fonction de $\mathrm{x}$ ) pour $\mathrm{PbZr}_{0,52} \mathrm{Ti}_{0,48} \mathrm{O}_{3}$. Cette explication a été remise en question par la découverte récente d'une phase monoclinique $\left(F_{M}\right.$ - groupe d'espace $\mathrm{Cm}$ ) autour de cette composition [3]. La direction de la polarisation dans cette phase se situe entre les directions $[001]_{\mathrm{Q}}$ et $[111]_{\mathrm{R}}$ et cela pourrait expliquer la forte réponse piézoélectrique [4]. Très récemment, une seconde phase monoclinique stable à basse température $\left(F_{M}\right.$ - groupe d'espace $\left.C c\right)$ a été mise en évidence [5]. Cette phase est obtenue à partir de la phase $F_{M}$ $(\mathrm{Cm})$ par rotation des octaèdres $(\mathrm{Zr}, \mathrm{Ti}) \mathrm{O}_{6}$ avec, comme conséquence, le doublement de la maille.

\section{PARTIE EXPERIMENTALE}

L'étude de solides sous haute pression présente des problèmes particuliers (petite taille de l'échantillon, forte contribution de la cellule haute pression etc.). Il est donc souvent très avantageux de recourir à plusieurs techniques complémentaires comme dans cette étude sur PZT. Les techniques souvent utilisées pour étudier la matière sous haute pression sont les suivantes [6-8] : diffraction des rayons X, diffraction des neutrons, EXAFS, spectroscopie infrarouge, diffusion Raman, diffusion Brillouin, ATD, mesures de résistivité, mesures diélectriques. Les quatre techniques expérimentales qui ont servi pour l'étude du diagramme de phases de PZT sont présentées ci-dessous.

\subsection{Synthèse des solutions solides PbZr1-xTixO3}

Les solutions solides de $\mathrm{Pb}\left(\mathrm{Zr}_{1-\mathrm{x}} \mathrm{Ti}_{\mathrm{x}}\right) \mathrm{O}_{3}(\mathrm{x}=0,48,0,50,0,52,0,56,0,60,0,80)$ ont été synthétisées à l'état solide par réactions en phase solide à partir d'oxydes de très haute pureté $(>99,9 \%)[9,10]$. La pureté et l'homogénéité des échantillons ont été contrôlées par cartographie micro-Raman et par

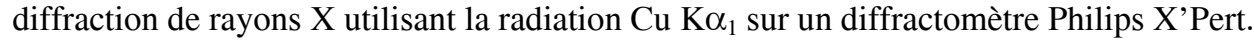

\subsection{Diffusion Raman}

Les spectres Raman hautes pressions ont été enregistrés en utilisant une cellule à enclumes de diamant (CED) à membrane couplée à un spectromètre Jobin-Yvon U 1000 équipé d'un CCD refroidi à l'azote liquide. Les expériences décrites ont été effectuées en rétro diffusion avec la radiation de l'ion Krypton à $647,1 \mathrm{~nm}$ afin de s'affranchir des vibrations de surfaces. Les échantillons sous forme de poudre ont été introduits dans un joint métallique avec un cristal de rubis et de l'argon comme milieu transmetteur de pression. Ainsi, la pression induite est estimée en se basant sur le déplacement de la raie de fluorescence $\mathrm{R}_{1}$ du rubis [11] en utilisant l'équation suivante :

$$
P\left(\lambda_{R 1}\right)=248,4 \mathrm{GPa}\left[\left(\frac{\lambda_{R 1}(P)}{\lambda_{R 1}(0)}\right)^{7,665}-1\right]
$$

\subsection{Diffraction des rayons $\mathrm{X}$}

L'étude par diffraction de rayons X sous pression a été effectuée en dispersion angulaire avec une source classique (tube à foyer fin - anticathode de Mo) et un écran photostimulable en utilisant une CED. Un mélange 4:1 méthanol : éthanol ou 21:4: 1 méthanol : éthanol : eau servait de milieu transmetteur de pression. Les marqueurs de pression utilisés étaient le rubis [11] (voir section 2.2) ou 
$\mathrm{NaCl}$ [12]. Pour ce dernier, la pression est calculée à partir du volume déduit des résultats de diffraction des rayons $\mathrm{X}$ en utilisant une équation d'état Birch-Murnaghan (équation 3) avec les valeurs suivantes : $V_{0}=0,17943 \mathrm{~nm}^{3}, B_{0}=23,635 \mathrm{GPa}, B_{0}^{\prime}=4,919, B^{\prime \prime}{ }_{0}=-0,0025$. Les affinements Rietveld ont été effectués avec le programme FULLPROF [13].

\subsection{Diffraction des neutrons}

Les expériences de diffraction neutronique ont été réalisées à la source de spallation ISIS (Chilton, G. B.) sur les instruments POLARIS et PEARL en utilisant une presse Paris-Edimbourg. Un mélange 4 : 1 méthanol : éthanol deutérié servait de milieu transmetteur de pression. La pression a été déterminée en utilisant les équations d'état de PZT obtenues préalablement à partir de l'étude par diffraction des rayons X. Les structures des différentes phases ont été affinées grâce au programme GSAS [14].

\subsection{Mesures diélectriques}

Les mesures diélectriques en fonction de la fréquence $(5 \mathrm{kHZ}-1 \mathrm{MHz})$ ont été effectuées avec un pont d'impédance Agilent 4294A. Des pastilles céramiques de PZT (98\% densité) ont été placées au troisième étage d'un compresseur Unipress. L'essence F servait de milieu transmetteur de pression.

\section{RÉSULTATS}

Les études $[9,10]$ sur la composition morphotropique $(\mathrm{x}=0.48)$ montrent que $\mathrm{PbZr}_{0,52} \mathrm{Ti}_{0,48} \mathrm{O}_{3}$ $\left(F_{M}-C m\right)$ subit une série de transformations de phases sous pression. La phase haute pression ( $\mathrm{P}>5 \mathrm{GPa}$ ) est pseudo-cubique. Toutefois, toutes les phases sont de basse symétrie (monoclinique ou triclinique). Ces résultats conjugués, obtenus par diffraction de rayons $\mathrm{X}$, diffraction neutronique, diffusion Raman et mesures diélectriques nous ont permis de proposer, dans un premier temps, un diagramme de phases $\mathrm{P}-\mathrm{T}$ pour $\mathrm{PbZr}_{0,52} \mathrm{Ti}_{0,48} \mathrm{O}_{3}$ [10]. Par la suite, les investigations ont été complétées par l'étude de compositions riches en $\mathrm{Ti}(\mathrm{x}=0,50,0,52,0,56,0,60,0,80)$ de symétrie quadratique à température et pression ambiantes. Là encore, des transitions de phases ont été mises en évidence par mesures diélectriques, diffraction des rayons $\mathrm{X}$ et diffusion Raman (Figure 2). Ainsi, dans le cas de $\mathrm{PbZr}_{0,40} \mathrm{Ti}_{0,60} \mathrm{O}_{3}$, une importante modification du spectre Raman se produit vers 1,5 GPa. A plus faible pression, $\mathrm{PbZr}_{0,40} \mathrm{Ti}_{0,60} \mathrm{O}_{3}$ se comporte comme $\mathrm{PbTiO}_{3}$, qui subit vers $12 \mathrm{GPa}$ une transition ferroélectrique (quadratique) - paraélectrique (cubique) classique avec un mode mou [15]. A partir de 1,5 GPa, $\mathrm{PbZr}_{0,40} \mathrm{Ti}_{0,60} \mathrm{O}_{3}$ présente un spectre avec des raies larges, comme pour la phase monoclinique de $\mathrm{PbZr}_{0,52} \mathrm{Ti}_{0,48} \mathrm{O}_{3}$, et exempt de modes mous. Une seconde transition de phase est mise en évidence grâce à l'apparition de nouvelles raies vers $3 \mathrm{GPa}$. Ces deux transitions sont également observées par diffraction de rayons $\mathrm{X}$ et correspondent à une diminution de la compressibilité dans le cas de la première et à un changement de volume pour la seconde. Pour ces compositions riches en titane, la constante diélectrique continue à augmenter jusqu'à $2 \mathrm{GPa}$ au lieu de diminuer comme dans le cas de $\mathrm{PbZr}_{0,52} \mathrm{Ti}_{0,48} \mathrm{O}_{3}$; les transitions se manifestent dans chaque cas par une rupture de pente sur les tracés $1 / \varepsilon_{\mathrm{R}}^{\prime}=\mathrm{f}(P)$. La structure de $\mathrm{PbZr}_{0,40} \mathrm{Ti}_{0,60} \mathrm{O}_{3}$ a été affinée à haute pression par diffraction neutronique. Les résultats confirment que la structure est monoclinique $F_{M}(\mathrm{Cm})$ à 2.7 et $F_{M}(\mathrm{Cc})$ à $4.1 \mathrm{GPa}$. Une phase pseudo-cubique paraélectrique (triclinique $F 1$ ) est obtenue à $10 \mathrm{GPa}$ via une autre phase ferroélectrique intermédiaire de symétrie triclinique $(F 1)$. La phase $F_{M}(C c)$ et les deux phases tricliniques sont caractérisées par une rotation des octaèdres $(\mathrm{Zr}, \mathrm{Ti}) \mathrm{O}_{6}$ qui s'amplifie avec la pression. Parallèlement, on observe une diminution des déplacements ferroélectriques. On retrouve, ici, deux des mécanismes de compression classiques, à savoir la rotation des polyèdres et l'augmentation de la coordinence du cation (la coordinence de $5+1$ dans les phases ferroélectriques devient régulière (coordinence de 6) dans la phase paraélectrique). 


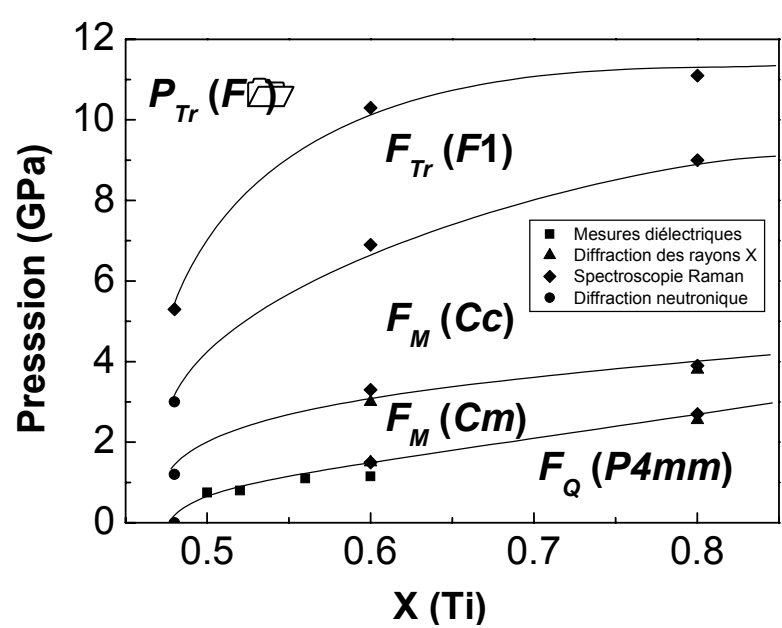

a)

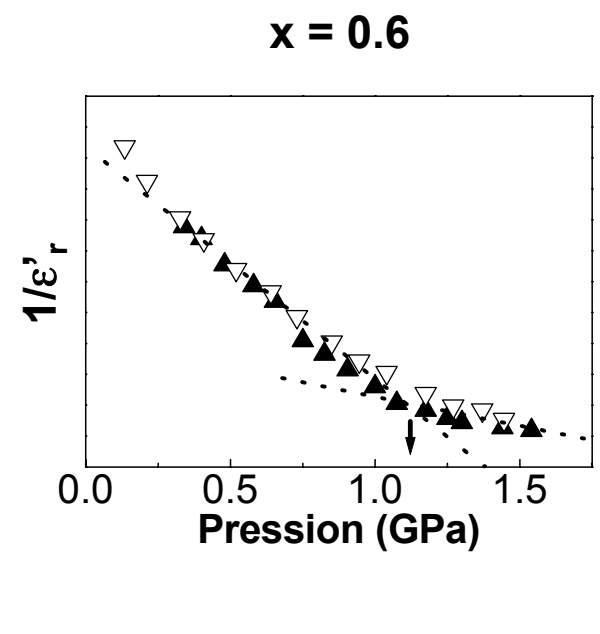

b)

Figure 2. a) Diagramme de phases $\mathrm{P}-\mathrm{x}(\mathrm{x}=0,48-0,80)$ de PZT b) mesures diélectriques $(100 \mathrm{MHz})$ pour $\mathrm{x}=0,60$ (symboles : $\boldsymbol{\Lambda}$ - compression ; $\nabla$ - décompression).

\section{CONCLUSIONS}

L'application d'une pression hydrostatique modifie la structure cristalline des solides avec pour conséquence un effet marqué sur la stabilité des phases. Les études menées sur la solution solide PZT et issues d'expériences conjointes par diffraction des rayons $X$, diffraction neutronique, diffusion Raman et mesures diélectriques sous pression, montrent que la phase monoclinique $F_{M}(\mathrm{Cm})$, qui est à l'origine des propriétés piézoélectriques remarquables de la composition morphotropique $\left(\mathrm{PbZr}_{0,52} \mathrm{Ti}_{0,48} \mathrm{O}_{3}\right)$ à pression ambiante, peut également être obtenue à des pressions modérées pour les solutions solides riches en $\mathrm{PbTiO}_{3}$. Dans la perspective d'applications sous forte contrainte (processus inévitable lié à la miniaturisation des dispositifs), le diagramme de phase $P$-x établi à partir de ces résultats devrait permettre d'optimiser l'élaboration de ces matériaux en «adaptant » la composition de la solution solide PZT en fonction des besoins.

\section{Remerciements}

Nous remercions F. Gorelli (LENS, Florence, Italie), L. Konczewicz (GES, Université Montpellier II), C. Bousquet (CEM2, Université Montpellier II), S. Hull, W. G. Marshall, D. Francis (ISIS, Chilton, G. B.) pour leurs contributions aux différentes expériences sur PZT.

\section{Références}

[1] Richet P., "Quelques aspects thermodynamiques de la pression" dans La pression : un outil pour les sciences, J. C. Chervin et J. Peyronneau Eds. (CNRS Editions, Paris, 2003) pp. 43-84

[2] Birch F., Phys. Rev. 71 (1947) 809-824

[3] Noheda B., Cox D.E., Shirane G., Gonzalo J.A., Cross L.E., et Park S.-E., Appl. Phys. Lett. 74, (1999) 2059-2061

[4] Guo R., Cross L.E., Park S.-E., Noheda B., Cox D.E., Shirane G., Phys. Rev. Lett. 84 (2000) 54235426 
[5] Hatch D.M., Stokes H.T., Ranjan R., Ragini, Mishra S.K., Pandey D., Kennedy B.J., Phys. Rev. B $\mathbf{6 5}$ (2002) 212101-1-4

[6] Ferraro, J.R., Vibrational spectroscopy at high external pressures (Academic Press, Orlando, 1984)

[7] Holzapfel W.B., Isaacs N.S. Eds., High-pressure techniques in chemistry and physics - a practical approach (Oxford University Press, Oxford, 1997) pp. 1-398

[8] Hazen R.M., Downs R.T. Eds., Rev. Mineral. Geochem. 41 (2000) 425-596

[9] Rouquette J., Haines J., Bornand V., Pintard M., Papet Ph., Astier R., Léger J.M., Gorelli F., Phys. Rev. B 65 (2002) 214102-1-4

[10] Rouquette J., Haines J., Bornand V., Pintard M., Papet Ph., Bonnet B., Gorelli F.A., Solid State Sci. 5 (2003) 451-457

[11] Mao H.K., Xu J., Bell P.M., J. Geophys. Res. 91 (1986) 4673-4676

[12] Decker D.L., J. Appl. Phys. 42 (1971) 3239-3244

[13]Rodríguez-Carvajal J., Physica B 192 (1993) 55-69

[14] Larson A.C., Von Dreele R.B., GSAS: General Structure Analysis System, (LANL, Los Alamos, 1994).

[15] Sanjurjo J. A., López-Cruz E., Burns G., Phys. Rev. B 28 (1983) 7260-7268 